Bull. Austral. Math. Soc.

VOL. 39 (1989) [335-338]

\title{
ON CONVERGENCE OF THE CONJUGATE FOURIER SERIES OF A FUNCTION OF WIENER'S CLASS
}

\author{
RAFAT N. SIDdiQI
}

The main object of this paper is to extend the theorem of W.H. Young on convergence of the conjugate Fourier series to Wiener's class. We prove that the conjugate Fourier series of a function of Wiener's class converges to $\bar{f}(x)$ for every $x$ for which $\bar{f}(x)$ exists.

\section{INTRODUCTION}

Let $f$ be a $2 \pi$-periodic function defined on $[0,2 \pi]$. We set

$$
\stackrel{b}{V}_{p}^{b}(f)=\sup \left\{\sum_{i=1}^{n}\left|f\left(t_{i}\right)-f\left(t_{i-1}\right)\right|^{p}\right\}^{1 / p} \quad(1 \leqslant p<\infty)
$$

where the supremum has been taken with respect to all partitions $P: a=t_{0}<t_{1}<$ $t_{2}<\ldots<t_{n}=b$ of any segment $[a, b]$ contained in $[0,2 \pi]$. We call $\underset{a}{V_{p}}(f)$ the $p$-th total variation of $f$ on $[a, b]$. If we denote the $p$-th total variation of $f$ on $[0,2 \pi]$ by $V_{p}(f)$, then we define Wiener's class simply by

$$
V_{p}=\left\{f \mid V_{p}(f)<\infty\right\}
$$

It is clear that $V_{1}$ is the ordinary class of functions of bounded variation, introduced by Jordan. The class $V_{p}$ was first introduced by Wiener in [4]. We note [3] that

$$
V_{p_{1}} \subset V_{p_{2}} \quad\left(1<p_{1}<p_{2}<\infty\right)
$$

is a strict inclusion. Hence for an arbitrary $(1<p<\infty)$, Wiener's class $V_{p}$ is strictly larger than the class $V_{1}$.

\section{Received 6 July 1988}

This research work was supported by the Grant No. SM054 of the College of Graduate Studies of Kuwait University.

Copyright Clearance Centre, Inc. Serial-fee code: 0004-9729/89 \$A2.00+0.00. 


\section{SOME KNOWN RESULTS}

Let $f \in V_{p}(1 \leqslant p<\infty)$ and let

$$
f(x) \sim \frac{a_{0}}{2}+\sum_{n=1}^{\infty}\left(a_{n} \cos n x+b_{n} \sin n x\right)
$$

be its Fourier series, then the series conjugate to (1) is given by

$$
\sum_{n=1}^{\infty}\left(a_{n} \sin n x-b_{n} \cos n x\right)
$$

We denote by $S_{n}(x)$ and $\overline{S_{n}}(x)$ the $n$-th partial sums of the series (1) and (2) respectively. We write

$$
\begin{aligned}
\psi_{x}(t) & =\frac{1}{2}\{f(x+t)-f(x-t)\} \\
\bar{f}(x) & =\lim _{\varepsilon \rightarrow 0^{+}} \bar{f}(x, \varepsilon)=\lim _{\varepsilon \rightarrow 0^{+}}\left\{\frac{-2}{\pi} \int_{\varepsilon}^{\pi} \frac{\psi_{x}(t)}{t \tan t / 2} d t\right\}
\end{aligned}
$$

and

$$
\overline{D_{n}}(t)=\sum_{k=1}^{n} \sin k t=\frac{\cos t / 2-\cos \left(n+\frac{1}{2}\right) t}{2 \sin t / 2}
$$

Wiener [4] proved that the Fourier series of a function of $V_{p}(1 \leqslant p<\infty)$ converges almost everywhere on $[0,2 \pi]$. Recently, we [2] strengthened the above result of Wiener by proving the following theorem.

Theorem A. If $f \in V_{p}(1 \leqslant p<\infty)$ then the Fourier series of $f$ converges to $\frac{1}{2}\{f(x+0)+f(x-0)\}$ at every $x \in[0,2 \pi]$.

There is also the following well-known theorem due to Young [5] (see Zygmund [6, p.59]) on convergence of the conjugate Fourier series of a function of bounded variation.

Theorem B. If $f \in V_{1}$, then the conjugate series (2) converges to $\bar{f}(x)$ at every $x \in[0,2 \pi]$, provided that $\bar{f}(x)$ exists.

There is a classical result of Privalov (see Zygmund [6, Chapter IV, Theorem 3.1]) which states that $\bar{f}(x)$ exists a.e. for any $f \in L^{1}$.

\section{The Main Result}

The main object of this paper is to extend Theorem $B$ into the strictly larger class $V_{p}$ for every $p$. To be precise, we give a simple proof of the following theorem. 
THEOREM. If $f \in V_{p}(1<p<\infty)$, then the conjugate series (2) converges to $\bar{f}(x)$ at every $x \in[0,2 \pi]$, for which $\bar{f}(x)$ exists.

Proof: We can write

$$
\overline{S_{n}}(x)=-\frac{2}{\pi} \int_{0}^{\pi} \psi_{x}(t) \overline{D_{n}(t)} d t
$$

hence

$$
\begin{aligned}
\overline{S_{n}}(x)-\bar{f}(x, \pi / n)=- & \frac{2}{\pi} \int_{0}^{\pi} \frac{\psi_{x}(t)}{2 \sin t / 2}\left(\cos t / 2-\cos \left(n+\frac{1}{2}\right) t\right) \\
& +\frac{2}{\pi} \int_{\pi / n}^{\pi} \frac{\psi_{x}(t)}{2 \tan t / 2} d t \\
=- & \frac{2}{\pi} \int_{0}^{\pi / n} \frac{\psi_{x}(t)}{2 \sin t / 2}\left(\cos t / 2-\cos \left(n+\frac{1}{2}\right) t\right) d t \\
& +\frac{2}{\pi} \int_{\pi / n}^{\pi} \frac{\psi_{x}(t)}{2 \sin t / 2} \cos \left(n+\frac{1}{2}\right) t d t \\
= & I_{1}+I_{2}, \quad \text { say. }
\end{aligned}
$$

Using Hölder's inequality (see Rudin [1]), we get

$$
\begin{aligned}
\left|I_{1}\right| & =\left|\frac{2}{\pi} \int_{0}^{\pi / n} \psi_{x}(t) \overline{D_{n}}(t) d t\right| \\
& \leqslant \frac{2}{\pi}\left\{\left(\int_{0}^{\pi / n}\left|\psi_{x}(t)-\psi_{x}(0)\right|^{p}\right)^{1 / p}\left(\int_{0}^{\pi / n}\left|\overline{D_{n}}(t)\right|^{q}\right)^{1 / q}\right\} \\
& \leqslant \frac{2}{\pi}\left(\int_{0}^{\pi / n}\left[V_{0}^{\pi / n}\left(\psi_{x}\right)\right]^{p} d t\right)^{1 / p}\left(\int_{0}^{\pi / n} n^{q} d t\right)^{1 / q} \\
& \leqslant 2 V_{0}^{\pi / n}\left(\psi_{x}\right) .
\end{aligned}
$$

But ${ }_{0}^{\psi} p\left(\psi_{x}\right)$ tends to zero as $n \rightarrow \infty$. Hence $I_{1} \rightarrow 0$ as $n \rightarrow \infty$.

Now consider the integral

$$
I_{2}=\frac{2}{\pi} \int_{\pi / n}^{\pi} \frac{\psi_{x}(t) \cos \left(n+\frac{1}{2}\right) t}{2 \sin t / 2} d t=-\frac{2}{\pi} \int_{\pi / n}^{\pi} \psi_{x}(t) d g(t)
$$

where

$$
g(t)=\int_{t}^{\pi} \frac{\cos \left(n+\frac{1}{2}\right) x}{2 \sin x / 2} d x
$$


Using integration by parts, we get

$$
\left|I_{2}\right| \leqslant \frac{2}{\pi}\left|\psi_{x}\left(\frac{\pi}{n}\right) g\left(\frac{\pi}{n}\right)\right|+\frac{2}{\pi} \int_{\pi / n}^{\pi}\left|g(t) d \psi_{x}(t)\right| .
$$

But by using the second mean value theorem, we get

$$
g(t)=\frac{1}{2 \sin t / 2} \int_{t}^{\xi} \cos \left(n+\frac{1}{2}\right) x \quad d x, \quad(t<\xi<\pi),
$$

hence

$$
|g(t)| \leqslant \frac{2}{n} \frac{1}{2 \sin t / 2} \leqslant \frac{\pi}{n t}
$$

From ( 7$)$ and (8) we obtain

$$
\left|I_{2}\right| \leqslant \frac{2}{\pi} V_{0}^{\pi / n}\left(\psi_{x}\right)+\frac{2}{n} \int_{\pi / n}^{\pi} \frac{\left|d \psi_{x}(t)\right|}{t} .
$$

But ${ }_{0}^{\pi / n}\left(\psi_{x}\right)$ tends to zero as $n \rightarrow \infty$, and by using Hölder's inequality (see Rudin [1]), we get

$$
\left|\int_{\pi / n}^{\pi} \frac{\left|d \psi_{x}(t)\right|}{t}\right| \leqslant\left(\int_{\pi / n}^{\pi}\left|\psi_{x}(t)-\psi_{x}(0)\right|^{p}\right)^{1 / p}\left(\int_{\pi / n}^{\pi} \frac{d t}{t^{q}}\right)^{1 / q}
$$

where $1 / p+1 / q=1$. Since $\left|\psi_{x}(t)-\psi_{x}(0)\right|^{p}$ is majorised by $\left[V_{p}\left(\psi_{x}\right)\right]^{p}$ and the integral $\int_{\pi / n}^{\pi}(d t) /\left(t^{q}\right)$ is convergent for $q>1$, hence we can find a positive constant $M$ independent of $n$ such that

$$
\left|I_{2}\right| \leqslant \frac{M}{n} V_{p}\left(\psi_{x}\right)
$$

which tends to zero as $n \rightarrow \infty$. This completes the proof of our theorem.

\section{REFERENCES}

[1] W.Rudin, Fourier Analysis On Groups (Interscience Publishers, New York, 1962).

[2] R.N. Siddiqi, 'A note on convergence of Fourier series of a function on Wiener's class', Canad. Math. Bull. 20 (2) (1977), 243-247.

[3] R.N. Siddiqi, 'Generalized absolute continuity of a function of Wiener's class', Bull. Austral. Math. Soc. 22 (2) (1980), 253-258.

[4] N. Wiener, 'The quadratic variation of a function and its Fourier coefficients', Mass. J. of Math. 3 (1924), 72-94.

[5] W.H. Young, 'Konvergenzbedingungen für die verwandte Reihe einer Fourier-schen Reihe', Münch. Ber. 41 (1911), 361-371.

[6] A. Zygmund, Trigonometric series, Vol. I (Cembridge University Press, 1959).

\section{Department of Mathematics}

P.O. Box 5969

Kuwait University

Kuwait 